\title{
A Phase II Study of Antineoplastons A10 and AS2-1 in Children with High-Grade Glioma. Final Report (Protocol BT-06), and Review of Recent Trials
}

\author{
Stanislaw R. Burzynski*, Tomasz J. Janicki, Gregory S. Burzynski, Ania Marszalek \\ Burzynski Clinic, Houston, USA \\ Email: ${ }^{*}$ srb@burzynskiclinic.com
}

Received 18 April 2014; revised 10 May 2014; accepted 18 May 2014

Copyright (C) 2014 by authors and Scientific Research Publishing Inc. This work is licensed under the Creative Commons Attribution International License (CC BY). http://creativecommons.org/licenses/by/4.0/

(c) (1) Open Access

\section{Abstract}

Standard treatment for high-grade glioma involves surgical resection followed by radiation therapy and temozolomide. Unfortunately, there are no standard treatment recommendations after recurrence and new therapies are needed for patients whose tumor recurs after first-line treatment. This single-arm, two-stage, interventional Phase II study evaluated the efficacy and safety of a combination of antineoplastons A10 and AS2-1. Nineteen patients were enrolled in the study (safety population), but fifteen patients with a median age of 9.4 years who met eligibility criteria were evaluated. The majority of subjects $(12 / 15)$ were Caucasian and $8 / 15(53 \%)$ were female. More than half $(53 \%)$ of patients were diagnosed with glioblastoma and $33 \%$ with anaplastic astrocytoma. All patients had failed standard therapy including surgery, radiation, and chemotherapy. Antineoplastons were administered intravenously every four hours (median dose of A10 6.9 $\mathrm{g} / \mathrm{kg} / \mathrm{d}$ and AS2-1 $0.30 \mathrm{~g} / \mathrm{kg} / \mathrm{d}$ ) until objective response was documented and thereafter for a further 8 months. Clinical evaluations were performed every 8 weeks. All patients enrolled in the study were included in the safety analysis but only patients fulfilling the inclusion criteria were included in the efficacy evaluation. The duration of treatment with antineoplastons ranged from 2 weeks to 120 weeks. A complete response was documented in 2/15 (13\%), partial response in $2 / 15(13 \%)$, stable disease in 3/15 (20\%). Progression-free survival at six months was $47 \%$ and overall survival $(O S)$ at one year was $33.3 \%$. One patient $(6.7 \%)$ survived 10 years from treatment start. A small group of patients suffered reversible Grade 3 and 4 toxicities including hypernatremia 2/19 (11\%) and decrease of neutrophils $1 / 19(5 \%)$. There were no chronic toxicities. There was improvement of quality of life in patients who had objective response. It is concluded that antineoplastons show efficacy with an acceptable profile in this cohort of patients with recurrent high-grade glioma.

${ }^{*}$ Corresponding author.

How to cite this paper: Burzynski, S.R., et al. (2014) A Phase II Study of Antineoplastons A10 and AS2-1 in Children with High-Grade Glioma. Final Report (Protocol BT-06), and Review of Recent Trials. Journal of Cancer Therapy, 5, $565-577$. 


\section{Keywords}

\section{Antineoplastons A10 and AS2-1, Glioblastoma, Phase II Clinical Trial, Recurrent Glioma}

\section{Introduction}

While research carried out in recent years has brought exciting new advances in the field of neuro-oncology, this still needs to be translated into an improvement in clinical results [1]. The standard of care for newly diagnosed high-grade glioma (HGG) is surgical resection followed by daily temozolomide (TMZ) and radiation therapy (RT) with subsequent adjuvant TMZ [2] [3]. Unfortunately, there are no standard therapy recommendations after recurrence [3]. The reported results from studies with currently available treatments are even more disappointing in pediatric HGG, and very little progress has been made over the past decade regarding the introduction of effective therapeutic regimens [4]. HGG in children are relatively uncommon and constitute approximately $17 \%$ of all pediatric brain tumors [5]. Glioblastoma multiforme (GBM) is less common than anaplastic astrocytoma (AA). In children below 15 years of age GBM constitute only $2.8 \%$ of all central nervous system (CNS) tumors [5].

Antineoplastons (ANP) A10 and AS2-1 are synthetic amino acid derivatives. A10 is a formulation consisting of a 4:1 ratio of phenylacetylglutaminate sodium (PG) and phenylacetylisoglutaminate sodium (isoPG). AS2-1 is a formulation with a 4:1 ratio of phenylacetate sodium (PN) and PG [6]. Initial encouraging clinical responses in the treatment of HGG led to the design of this single-arm Phase II study to assess the efficacy and safety of ANP therapy in children diagnosed with HGG [6]-[11].

\section{Patients and Methods}

\subsection{Patient Population}

Patients, all aged less than 18 years and more than six months of age with a diagnosis of incurable HGG (GBM, AA, disseminated astrocytoma) who had recurrent or progressive disease or who had residual tumor following standard therapy were recruited into the study. Patients were required to have evidence of brain tumor by gadolinium-enhanced magnetic resonance imaging (MRI) performed within two weeks prior to study enrollment. There were no exclusion criteria based on tumor size, multifocality or leptomeningeal involvement. Eligibility criteria also included a Karnofsky Performance Status (KPS) of 60 - 100, but additional patients with KPS below 50 could be accepted based on case-by-case evaluation and with exception granted by the FDA. The subjects were required to have relatively normal hematopoietic and hepatic function with white blood cell count (WBC) over $1500 \mathrm{~mm}^{3}$ and platelet count over $50,000 \mathrm{~mm}^{3}$, no evidence of hepatic or renal insufficiency, and a total bilirubin and serum creatinine of no higher than $2.5 \mathrm{mg} / \mathrm{dL}$ and SGOT and SGPT no higher than $5 \mathrm{x}$ the upper limits. At least eight weeks must have relapsed since the last dose of RT, and at least four weeks since the last dose of chemotherapy (six weeks for nitrosoureas), or immunotherapy. The use of corticosteroids was permitted to reduce symptoms and signs attributed to cerebral edema, but it was recommended that the smallest doses compatible with the preservation of optimal neurologic function, be used. Confirmation of the pathologic diagnosis by an outside pathologist was also required. The exclusion criteria included brainstem location of the tumor, serious active infection, fever or other serious concomitant disease that would interfere with the evaluation of the treatment (e.g., severe heart or lung disease).

Patients were removed from the study if 1) progressive disease (PD) developed after at least 4 weeks of ANP therapy 2) if toxicity levels became unacceptable, 3 ) if the subject developed a concurrent illness that interfered with therapy, 4) if the subject or guardian asked to be removed from the study or became non-compliant with the study criteria, and 5) if the subject completed at least 8 months of treatment after determination of complete response (CR), partial response (PR) or stable disease (SD).

All study subjects and/or guardians read, understood, and signed written informed consent prior to enrollment. This study was conducted in accordance with the US Code of Federal Regulations, Title 21, Parts 11, 50, 56, and 312; the Declaration of Helsinki (1964) including all amendments and revisions; the Good Clinical Practices: Consolidated Guideline (E6); International Conference on Harmonization; and the FDA's Guidance for Industry. 
The study was sponsored by the Burzynski Research Institute Inc. (BRI) and conducted by the Burzynski Clinic (BC) in Houston, Texas. The patients did not pay for the investigational agents.

\subsection{Study Design}

The study was designed as a single-arm, two-stage, interventional Phase II trial of ANP as the monotherapy in a high-risk, poor-prognosis study population [12]. The study was listed by the National Cancer Institute (NCI) and the protocol was initially drawn up by the NIH and later transferred to the BRI and BC. It was supervised by the independent Institutional Review Board (BRI-IRB, BC-BT-06).

The study was performed according to Protocol BT-06 which was submitted to the FDA under the IND 43,742. The study commenced on August 24, 1993 and closed to accrual on May 19, 2005. Subsequently, the protocol was amended by BRI several times; however, none of the amendments altered the aim or design of the original study objectives/outcomes.

\section{Statistical Consideration}

The sample size was calculated based upon the method described by Chang et al. [12]. A response rate to ANP of $\geq 10 \%$ was considered "of interest", and the primary endpoint was to determine the overall response rate (confirmed CR or PR) to ANP therapy. An interim analysis would be conducted after 20 subjects had enrolled in the study. If one or more patients achieved a confirmed radiographic response, an additional 20 subjects would be recruited. Survival was measured from the first day of ANP administration until death from any cause, and time to treatment failure was likewise measured from the first day of the treatment until the date of first observation of progressive disease or death from any cause whichever came first. The distributions of survival and treatment failure were estimated by Kaplan-Meier analysis.

\section{Treatment}

The median maximum effective daily dose (dose which accomplished beginning of objective response (OR; CR + PR) or determination of SD) of ANP A10 ranged from 1.29 to $15.23 \mathrm{~g} / \mathrm{kg} / \mathrm{d}$ with a median of $9.56 \mathrm{~g} / \mathrm{kg} / \mathrm{d}$. For AS2-1, the median maximum effective daily dose was $0.30 \mathrm{~g} / \mathrm{kg} / \mathrm{d}$, with a range of 0.24 to $0.48 \mathrm{~g} / \mathrm{kg} / \mathrm{d}$. The median maximum effective daily dose of A10 in subjects with an OR was $9.5 \mathrm{~g} / \mathrm{kg} / \mathrm{d}$ (range 1.29 to $15.23 \mathrm{~g} / \mathrm{kg} / \mathrm{d}$, standard deviation $=6.12 \mathrm{~g} / \mathrm{kg}, 95 \% \mathrm{CI}=2.89-14.87$ ) and for subjects with a SD response was $9.56 \mathrm{~g} / \mathrm{kg} / \mathrm{d}$ (range 8.35 to $10 \mathrm{~g} / \mathrm{kg} / \mathrm{d}$, standard deviation $=0.85 \mathrm{~g} / \mathrm{kg}, 95 \% \mathrm{CI}=8.34-10.27$ ). The median maximum effective dose of AS2-1 of subjects with an OR response was $0.36 \mathrm{~g} / \mathrm{kg} / \mathrm{d}$ (range 0.26 to $0.48 \mathrm{~g} / \mathrm{kg} / \mathrm{d}$, standard deviation = $0.11 \mathrm{~g} / \mathrm{kg}, 95 \% \mathrm{CI}=0.26-0.47$ ) and for subjects responding with a SD was $0.30 \mathrm{~g} / \mathrm{kg} / \mathrm{d}$ (range 0.24 to 0.42 $\mathrm{g} / \mathrm{kg} / \mathrm{d}$, standard deviation $=0.09 \mathrm{~g} / \mathrm{kg}, 95 \% \mathrm{CI}=0.22-0.42$ ). The duration of IV ANP therapy ranged from 2 to 120 weeks with a median of 21.9 weeks. Four subjects had OR and therapy ended after median of 53 weeks. One patient halted the treatment due to patient request. Six subjects halted therapy due to PD confirmed by MRI. Seven subjects halted the treatment due to worsening in clinical condition. One additional subject died within 24 hours of the last dose of ANP.

ANP A10 and AS2-1 were delivered via a dual-channel infusion pump and single-lumen subclavian catheter (Broviac or Groshong) every 4 hours. On the first day of administration of ANP the flow rate of the pump was maintained at $25 \mathrm{~mL} / \mathrm{h}$. Beginning from the second day, individual injections were given at 50 to $250 \mathrm{~mL} / \mathrm{h}$ depending on the patient's age and tolerance.

Approximate guidelines for flow rates are provided below:

1) Six months to two years old - flow rate $50 \mathrm{~mL} / \mathrm{h}$.

2) Two to four years old-flow rate $75 \mathrm{~mL} / \mathrm{h}$.

3) Four to seven years old —flow rate $100 \mathrm{~mL} / \mathrm{h}$.

4) Seven to ten years old-flow rate $150 \mathrm{~mL} / \mathrm{h}$.

5) Ten to sixteen years old-flow rate $200 \mathrm{~mL} / \mathrm{h}$.

6) Sixteen to eighteen years old-flow rate $250 \mathrm{~mL} / \mathrm{h}$.

The details of ANP therapy were published before [10]. The rationale for using two formulations of ANP was based on prior clinical trials, pharmacokinetic studies and laboratory research [6] [7]. The escalation of the dosage of ANP was required based on the results of prior studies carried out to determine whether patients were 
able to tolerate large volume infusions of intravenous fluids associated with higher doses of ANP [6]. As a safety precaution it is recommended that the escalation of the dosages will continue through Phase II and Phase III trials programs.

Medications that were considered necessary for the subjects' welfare and that did not interfere with the evaluation of treatment were given at the discretion of the investigator. The use of corticosteroids was carefully monitored. Treatment with other antineoplastic or immunomodulatory agents was not permitted during the study. Subjects received full supportive care, including transfusions of blood products and antibiotics when appropriate. No other anticancer mediation was permitted.

The initial three weeks of therapy were administered by BC staff on an outpatient basis, in Houston, Texas. The treatment did not require hospitalization. Subjects and/or their legal guardians were trained by clinic staff to self-administer ANP therapy during this time. Starting on week 4, ANP therapy was administered at home with 24-hour support available via phone or email. Treatment and monitoring of the subject's condition, once released to self-administered therapy, continued under the supervision of the subject's local physician.

\section{Evaluation and Follow-Up}

Prior to the start of treatment, a gadolinium-enhanced MRI measured all contrast-enhancing lesions. The products of the two greatest perpendicular diameters of all lesions were calculated and totaled, providing a baseline evaluation for each study subject. As a common practice at that time in other clinical trials, the tumor measurements were based on contrast enhanced lesions, but the overall tumor size was also measured including T2 and FLAIR images [13] [14]. The baseline provided the reference for determining response outcomes to the treatment. Blood and urine tests (complete blood count with differential, platelet count, reticulocyte count, and serum chemistry) anticonvulsant serum levels, prothrombin time, and partial thromboplastin time were carried out on all subjects prior to the start of treatment to establish normal baselines. The additional pretreatment measurements included KPS, vital signs, clinical disease status, demographics, medical history and current medications, physical examination with neurologic emphasis, chest X-rays and EKG. Toxicity was evaluated in all patients enrolled in the study (safety population). Data on adverse drug experiences (ADEs) were collected during the initial 3 weeks of ANP therapy by clinic staff at the BC. MRIs were repeated at least every 8 weeks during the first 2 years unless the patient's condition or confirmation of response required MRI within 4 weeks. Positron emission tomography (PET) scans were performed as necessary. When study subjects transitioned to homebased therapy administration, clinic staff made daily telephone contact for the first two months to ensure protocol compliance, to resolve any issues with therapy administration, and to continue assessing ADEs. Weekly contact was made starting in third month. Continued patient treatment with ANP was determined on a weekly basis and based upon the trial protocol, patient health status, and the response to treatment. The ADEs were graded according to the Common Terminology Criteria for Adverse Events (CTCAE v.3). Pharmacokinetic studies had been carried out in earlier Phase I and other Phase II studies and were not included in the study. Based on prior study there was no indication of interference with some medications, in particular, anti-seizure drugs.

\section{Results}

Subject enrollment started August 24, 1993, and continued through May 19, 2005. As of June 16, 2006, all subjects were removed from the therapy whether due to a subject request, OR, PD, or worsening clinical condition. The 15 patients who met eligibility criteria had a median age of 9.4 years. There was equal distribution between genders (53\% female, $47 \%$ male), and the majority of subjects (80\%) were Caucasian. Two subjects presented with disseminated disease in the brain and spinal cord, and three had thalamic location of the tumor. One patient had GBM/diffuse intrinsic pontine glioma (DIPG). The majority of patients had pathologic diagnoses of GBM, and the duration of neurological symptoms for over 6 months prior to enrollment to the study. 88\% of GBM patients underwent partial resection of the tumor and $75 \%$ failed RT. All of the GBM patients failed chemotherapy of which $62.5 \%$ underwent one regimen, $12.5 \%$ underwent treatment with two regimens, $12.5 \%$ with three regimens, and $12.5 \%$ with five consecutive regimens of chemotherapy.

Two out of five anaplastic astrocytoma patients underwent partial tumor resection. Two AA patients underwent one type of radiation and a single AA case received two types of radiation. All AA patients failed chemotherapy. The case of atypical teratoid rhabdoid tumor (AT/RT) was treated with surgery, hyper-fractionated ra- 
diation and chemotherapy, and had a persistent tumor. A single patient with disseminated grade II astrocytoma relapsed after radiation therapy.

Demographics for the subjects are summarized in Table 1. Patient demographics did not change during the study, and were similar to the other studies on pediatric HGG.

The trial enrolled 19 study subjects. Three patients were deemed ineligible because of brainstem location of the primary tumor, and one patient was ineligible because of lack of baseline MRI. These four patients were also non-evaluable since they did not have follow-up MRIs. Seven patients received exceptions from the FDA since their KPS were below 60 (two had KPS of 40 and 5 had KPS of 50). An additional patient received FDA exception because she was diagnosed with AT/RT and had a KPS of 50. Another patient received exception because of the diagnosis of GBM with brainstem involvement, and another patient because of the diagnosis of GBM and osteogenic sarcoma of the right-upper extremity.

\section{Responses and Survival}

The patient's diagnoses, recurrences and responses were confirmed by outside pathologists and radiologists (Table 2).

Table 1. Study population demographics—protocol BT-06.

\begin{tabular}{|c|c|}
\hline Characteristic & $\begin{array}{l}\text { Pediatric patients, } \\
\quad n=15(\%)\end{array}$ \\
\hline \multicolumn{2}{|l|}{ Age (years) } \\
\hline Median & 9.4 \\
\hline Range & $1-17.5$ \\
\hline \multicolumn{2}{|l|}{ Sex } \\
\hline Male & $7(47)$ \\
\hline Female & $8(53)$ \\
\hline Ethnicity & $\begin{array}{l}12 \mathrm{C}(80), 1 \mathrm{H}(7), \\
1 \mathrm{~A}(7), 1 \mathrm{O}(7)\end{array}$ \\
\hline \multicolumn{2}{|c|}{ Tumor Type } \\
\hline Glioblastoma multiforme & $8(53)$ \\
\hline Anaplastic astrocytoma & $5(33)$ \\
\hline Astrocytoma, grade II, disseminated & $1(7)$ \\
\hline Atypical teratoid rhabdoid tumor & $1(7)$ \\
\hline \multicolumn{2}{|c|}{ Clinical Characteristics } \\
\hline \multicolumn{2}{|l|}{ Duration of symptoms prior enrollment } \\
\hline$<6$ months $(\%)$ & $2(13)$ \\
\hline >6 months $(\%)$ & $13(87)$ \\
\hline \multicolumn{2}{|c|}{ Prior Treatment } \\
\hline Surgery & $10(67)$ \\
\hline \multicolumn{2}{|l|}{ Radiation Therapy } \\
\hline One regimen & $11(73)$ \\
\hline Two regimens & $1(7)$ \\
\hline \multicolumn{2}{|l|}{ Chemotherapy } \\
\hline Regimen 1 & $11(73)$ \\
\hline Regimen 2 & $1(7)$ \\
\hline Regimen 3 & $1(7)$ \\
\hline Regimen 5 & $1(7)$ \\
\hline Biologics & $2(13)$ \\
\hline Targeted Therapy & $1(7)$ \\
\hline
\end{tabular}

A-African American, C-Caucasian, H-Hispanic, O-Oriental. 
Table 2. Confirmation of diagnosis, recurrence and response.

\begin{tabular}{|c|c|c|c|c|c|c|c|c|c|c|c|c|}
\hline \multicolumn{5}{|c|}{ Confirmation of Diagnosis } & \multirow[b]{3}{*}{ 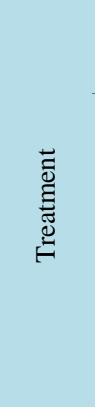 } & \multirow{2}{*}{\multicolumn{2}{|c|}{$\begin{array}{l}\text { Confirmation of } \\
\text { Recurrence }\end{array}$}} & \multirow{2}{*}{\multicolumn{2}{|c|}{$\begin{array}{l}\text { Confirmation of } \\
\text { Response to ANP }\end{array}$}} & \multirow{2}{*}{\multicolumn{3}{|c|}{ Tumor Measurements }} \\
\hline \multicolumn{3}{|c|}{ Pathology } & \multicolumn{2}{|c|}{ Radiology } & & & & & & & & \\
\hline : & 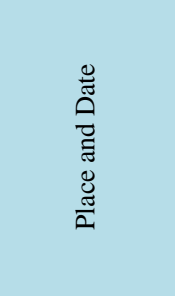 & 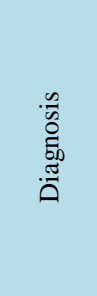 & 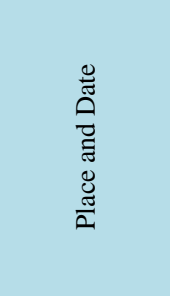 & 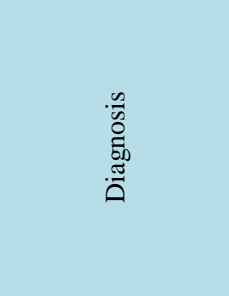 & & 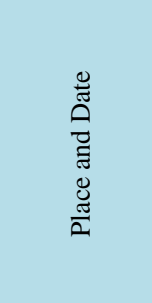 & 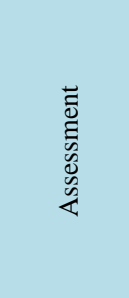 & 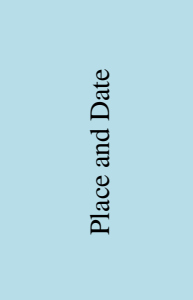 & 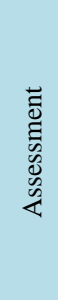 & \multicolumn{2}{|c|}{ 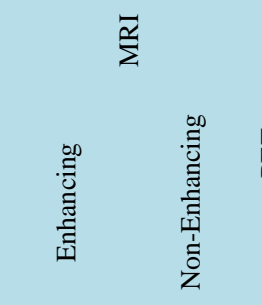 } & $\stackrel{-1}{a}$ \\
\hline 1 & $\begin{array}{c}\text { University } \\
\text { Hospital, } \\
\text { February 9, } \\
1993\end{array}$ & AT/RT & $\begin{array}{c}\text { University } \\
\text { Hospital, } \\
\text { January 9, } \\
1993\end{array}$ & $\begin{array}{c}\text { Right lateral } \\
\text { ventricle tumor } \\
\text { with spinal cord } \\
\text { and leptomeningeal } \\
\text { dissemination }\end{array}$ & $\begin{array}{l}\text { S RT } \\
\text { C }\end{array}$ & $\begin{array}{l}\text { University } \\
\text { Hospital, } \\
\text { August 2, } \\
1993\end{array}$ & $\begin{array}{l}\text { Persistent } \\
\text { tumor }\end{array}$ & $\begin{array}{c}\text { External } \\
\text { radiology CRR } \\
\text { May 14, 2003; } \\
\text { University } \\
\text { Hospital } \\
\text { Autopsy report } \\
\text { September 22, } \\
1995\end{array}$ & PR & $\begin{array}{l}\text { Decreased } \\
>50 \% \\
\text { NVT }\end{array}$ & Decreased & \\
\hline 2 & $\begin{array}{l}\text { University } \\
\text { Hospital, } \\
\text { February 10, } \\
1994\end{array}$ & GBM & $\begin{array}{c}\text { University } \\
\text { Hospital, } \\
\text { January 11, } \\
1994\end{array}$ & $\begin{array}{l}\text { Left parietal } \\
\text { lobe tumor }\end{array}$ & $\begin{array}{l}\text { S RT } \\
\text { C }\end{array}$ & $\begin{array}{c}\text { External } \\
\text { radiology, } \\
\text { January 4, } \\
1995\end{array}$ & $\begin{array}{l}\text { Persistent } \\
\text { tumor }\end{array}$ & $\begin{array}{l}\text { External } \\
\text { radiology, } \\
\text { CRR } \\
\text { November 19, } \\
2000\end{array}$ & PD & $\begin{array}{l}\text { Increased } \\
>50 \%\end{array}$ & Increased & \\
\hline 3 & $\begin{array}{c}\text { Regional } \\
\text { Cancer } \\
\text { Institute, } \\
\text { July 31, } 1995\end{array}$ & AA & $\begin{array}{c}\text { Regional } \\
\text { Cancer } \\
\text { Center, July } \\
1999\end{array}$ & $\begin{array}{l}\text { Left } \\
\text { parieto-occipital } \\
\text { tumor }\end{array}$ & $\begin{array}{l}\text { S RT } \\
\text { C }\end{array}$ & $\begin{array}{l}\text { Regional } \\
\text { Cancer } \\
\text { Center, } \\
\text { March 14, } \\
1996\end{array}$ & $\begin{array}{l}\text { Persistent } \\
\text { tumor }\end{array}$ & $\begin{array}{l}\text { External } \\
\text { radiology, } \\
\text { August 15, } \\
2000\end{array}$ & CR & NVT & Decreased & \\
\hline 4 & $\begin{array}{c}\text { University } \\
\text { Hospital, } \\
\text { January 31, } \\
1996\end{array}$ & GBM & $\begin{array}{c}\text { University } \\
\text { Hospital, } \\
\text { January 31, } \\
1996\end{array}$ & Thalamic tumor & $2 \mathrm{~S} 2 \mathrm{C}$ & $\begin{array}{l}\text { University } \\
\text { Hospital, } \\
\text { July 23, } \\
1996\end{array}$ & $\begin{array}{l}\text { Recurrent } \\
\text { tumor }\end{array}$ & $\begin{array}{c}\text { University } \\
\text { Hospital, } \\
\text { October 1, } \\
1996\end{array}$ & PD & Increased & Increased & \\
\hline 5 & $\begin{array}{l}\text { University } \\
\text { Hospital, } \\
\text { May 31, } \\
1996\end{array}$ & GBM & $\begin{array}{c}\text { University } \\
\text { Hospital, } \\
\text { May 21, } 1996\end{array}$ & $\begin{array}{l}\text { Right frontal } \\
\text { lobe tumor }\end{array}$ & S C B & $\begin{array}{l}\text { University } \\
\text { Hospital, } \\
\text { August 28, } \\
1996\end{array}$ & $\begin{array}{l}\text { Recurrent } \\
\text { tumor }\end{array}$ & $\begin{array}{l}\text { No follow-up } \\
\text { scans }\end{array}$ & $\mathrm{NE}$ & & & \\
\hline 6 & $\begin{array}{c}\text { Cancer } \\
\text { Institute, } \\
\text { July 18, } \\
1996\end{array}$ & A2 & $\begin{array}{c}\text { Cancer } \\
\text { Institute, } \\
\text { June 10, } 1996\end{array}$ & $\begin{array}{l}\text { Cervicomedullary } \\
\text { tumor extending to } \\
\text { the thoracic spinal } \\
\text { cord with } \\
\text { metastases }\end{array}$ & $\begin{array}{c}\text { Bx RT } \\
\text { B }\end{array}$ & $\begin{array}{c}\text { External } \\
\text { radiology, } \\
\text { April 24, } \\
1997\end{array}$ & $\begin{array}{l}\text { Recurrent } \\
\text { tumor }\end{array}$ & $\begin{array}{l}\text { No follow-up } \\
\text { scans }\end{array}$ & $\mathrm{NE}$ & & & \\
\hline 7 & $\begin{array}{c}\text { University } \\
\text { Hospital, } \\
\text { May 27, } 1998\end{array}$ & AA & $\begin{array}{c}\text { University } \\
\text { Hospital, } \\
\text { April } 161997\end{array}$ & $\begin{array}{l}\text { Right parietal } \\
\text { tumor }\end{array}$ & $2 \mathrm{Bx} \mathrm{C}$ & $\begin{array}{l}\text { University } \\
\text { Hospital, } \\
\text { May 16, } \\
1997\end{array}$ & $\begin{array}{l}\text { Persistent } \\
\text { tumor }\end{array}$ & $\begin{array}{c}\text { External } \\
\text { radiology } \\
\text { CRR, July 8, } \\
2000\end{array}$ & PR & $\begin{array}{l}\text { Decreased } \\
>50 \%\end{array}$ & Decreased & \\
\hline 8 & $\begin{array}{c}\text { Regional } \\
\text { Medical } \\
\text { Center, } \\
\text { November 3, } \\
1995\end{array}$ & GBM & $\begin{array}{c}\text { Regional } \\
\text { Medical } \\
\text { Center, } \\
\text { November 1, } \\
1995\end{array}$ & Left parietal tumor & $\begin{array}{c}\text { Bx RT } \\
\text { 3C }\end{array}$ & $\begin{array}{c}\text { External } \\
\text { radiology, } \\
\text { August 5, } \\
1997\end{array}$ & $\begin{array}{l}\text { Recurrent } \\
\text { tumor }\end{array}$ & $\begin{array}{l}\text { No Follow-up } \\
\text { scans }\end{array}$ & $\mathrm{NE}$ & & & \\
\hline 9 & $\begin{array}{l}\text { University } \\
\text { Hospital, } \\
\text { October 22, } \\
1993\end{array}$ & GBM & $\begin{array}{c}\text { University } \\
\text { Hospital, } \\
\text { October } 1993\end{array}$ & $\begin{array}{l}\text { Tumor in the } \\
\text { right ventricle }\end{array}$ & $\begin{array}{l}\text { S RT } \\
\text { C }\end{array}$ & $\begin{array}{l}\text { External } \\
\text { radiology, } \\
\text { November } \\
10,1997\end{array}$ & $\begin{array}{l}\text { Recurrent } \\
\text { tumor }\end{array}$ & $\begin{array}{c}\text { External } \\
\text { radiology, } \\
\text { April 6, } 1998\end{array}$ & $\mathrm{PD}$ & $\begin{array}{l}\text { Increased } \\
>50 \%\end{array}$ & Increased & \\
\hline 10 & $\begin{array}{c}\text { Regional } \\
\text { Medical } \\
\text { Center, } \\
\text { March 6, } 2000\end{array}$ & GBM & $\begin{array}{l}\text { Regional } \\
\text { Medical } \\
\text { Center, } \\
\text { January 21, } \\
2010\end{array}$ & DIPG & $\begin{array}{l}\text { S RT } \\
\text { C }\end{array}$ & $\begin{array}{c}\text { External } \\
\text { radiology, } \\
\text { June } 7,2000\end{array}$ & $\begin{array}{l}\text { Persistent } \\
\text { tumor }\end{array}$ & $\begin{array}{c}\text { External } \\
\text { radiology, } \\
\text { August 10, } \\
2000 \\
\text { September 28, } \\
2000 \\
\text { November } 16 \text {, } \\
2000\end{array}$ & SD & $\begin{array}{l}\text { Decreased } \\
>25 \%\end{array}$ & Stable & \\
\hline
\end{tabular}


Continued

\begin{tabular}{|c|c|c|c|c|c|c|c|c|c|c|c|c|}
\hline 11 & $\begin{array}{c}\text { University } \\
\text { Hospital, } \\
\text { January 14, } \\
2000\end{array}$ & AA & $\begin{array}{c}\text { University } \\
\text { Hospital, } \\
\text { January 12, } \\
2000\end{array}$ & Thalamic tumor & $\begin{array}{c}\text { Bx RT } \\
\text { C }\end{array}$ & $\begin{array}{c}\text { External } \\
\text { radiology, } \\
\text { July 26, } \\
2000\end{array}$ & $\begin{array}{l}\text { Persistent } \\
\text { tumor }\end{array}$ & $\begin{array}{c}\text { External } \\
\text { radiology, } \\
\text { September 20, } \\
2000 \\
\text { November 9, } \\
2000 \\
\text { December 18, } \\
2000 \text { January } \\
10,2001\end{array}$ & SD & $\begin{array}{c}\text { Decreased } \\
>10 \%\end{array}$ & Stable & \\
\hline 12 & $\begin{array}{l}\text { University } \\
\text { Hospital, } \\
\text { December } \\
\text { 14, 2001, } \\
\text { July 5, 2002 }\end{array}$ & GBM & $\begin{array}{l}\text { External } \\
\text { radiology, } \\
\text { December 8, } \\
2001\end{array}$ & Frontal lobe tumor & $\begin{array}{c}2 \mathrm{~S} R \mathrm{RT} \\
\mathrm{C}\end{array}$ & $\begin{array}{c}\text { External } \\
\text { radiology, } \\
\text { October 25, } \\
2002\end{array}$ & $\begin{array}{l}\text { Recurrent } \\
\text { tumor }\end{array}$ & $\begin{array}{c}\text { External } \\
\text { radiology } \\
\text { CRR, June 15, } \\
2008\end{array}$ & PR & $\begin{array}{c}\text { Decreased } \\
>50 \%\end{array}$ & Decreased & \\
\hline 13 & $\begin{array}{l}\text { Regional } \\
\text { Medical Cen- } \\
\text { ter, August } \\
\text { 31, } 2001\end{array}$ & AA & $\begin{array}{c}\text { Regional } \\
\text { Medical } \\
\text { Center, } \\
\text { August 30, } \\
2001\end{array}$ & Thalamic tumor & $\begin{array}{l}\text { Bx 2S } \\
\text { RT C }\end{array}$ & $\begin{array}{l}\text { External } \\
\text { radiology, } \\
\text { November } \\
21,2002\end{array}$ & $\begin{array}{l}\text { Persistent } \\
\text { tumor }\end{array}$ & $\begin{array}{c}\text { External } \\
\text { radiology, } \\
\text { January 8, } \\
2002\end{array}$ & $\mathrm{PD}$ & $\begin{array}{c}\text { Increased } \\
>50 \%\end{array}$ & Increased & \\
\hline 14 & $\begin{array}{c}\text { Regional } \\
\text { hospital, } \\
\text { February 21, } \\
2002\end{array}$ & AA & $\begin{array}{l}\text { Regional } \\
\text { hospital, } \\
\text { February 14, } \\
2003\end{array}$ & $\begin{array}{c}\text { Thalamic and } \\
\text { hypothalamic } \\
\text { tumor }\end{array}$ & $\begin{array}{c}\mathrm{Bx} C \\
\mathrm{~T}\end{array}$ & $\begin{array}{c}\text { External } \\
\text { radiology, } \\
\text { February 17, } \\
2004\end{array}$ & $\begin{array}{c}\text { Persistent } \\
\text { tumor }\end{array}$ & $\begin{array}{c}\text { External } \\
\text { radiology, } \\
\text { April 27, 2004 } \\
\text { June 29, 2004 } \\
\text { November 6, } \\
2004 \\
\text { December } \\
30,2004 \\
\text { March 23, } \\
2005 \\
\text { August 16, } \\
2005 \\
\text { September 22, } \\
2005 \\
\text { November 17, } \\
2005 \\
\text { January 18, } \\
2006\end{array}$ & SD & Stable size & Stable & 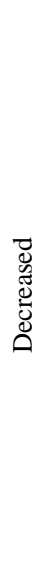 \\
\hline 15 & $\begin{array}{l}\text { University } \\
\text { Hospital, } \\
\text { May 3, } 2005\end{array}$ & GBM & $\begin{array}{l}\text { Regional } \\
\text { hospital, } \\
\text { September } \\
\text { 29, } 2004\end{array}$ & $\begin{array}{l}\text { Pineal region } \\
\text { tumor }\end{array}$ & $\begin{array}{l}\text { Bx S } \\
\text { RT 5C }\end{array}$ & $\begin{array}{c}\text { Cancer } \\
\text { Institute, } \\
\text { July 6, } 2005\end{array}$ & $\begin{array}{l}\text { Persistent } \\
\text { tumor }\end{array}$ & $\begin{array}{l}\text { No follow-up } \\
\text { scans }\end{array}$ & NE & & & \\
\hline
\end{tabular}

AA—anaplastic astrocytoma, A2—astrocytoma, grade 2, AT/RT—atypical teratoid/rhabdoid tumor, DIPG—diffuse intrinsic pontine glioma, GBM— glioblastoma multiforme, $\mathrm{CR}$ - complete response, $\mathrm{PR}$ - partial response, $\mathrm{PD}$-progressive disease, SD—stable disease, NE—non-evaluable, NVT— no visible tumor, C—chemotherapy, RT—radiation therapy, S—surgical resection, Bx—biopsy, B — biologic treatment, T—targeted therapy, CRR— central radiology review, MRI—magnetic resonance imaging, PET—positron emission tomography.

Out of 15 enrollees, two (13\%) achieved a CR, and an additional two had PR (13\%) (Figure 1 and Figure 2), three (20\%) had SD, four (27\%) had PD, and four (27\%) were not further analyzed because they did not have follow-up MRI.

They discontinued treatment proactively, and were withdrawn from the study as per subject's or guardian's request. It took a median of 83.6 weeks for a CR to be reached (range 66.9 to 100 weeks). The duration of SD was from 32 to 56 weeks. CR was determined in single cases of AT/RT and AA, PR was determined in single cases of AA and GBM. Three cases of SD included DIPG/GBM, and two cases of AA of thalamic location. The responses and survival data in the study subjects are presented in Table 3 and compared to the other studies in HGG. One patient diagnosed with AA of the thalamus and hypothalamus survived ten years since the treatment start. After she obtained SD and withdrew from the study, she was treated with combination chemotherapy and surgery. Post-treatment KPS increased in 4 patients (27\%), was stable in 7 patients (47\%) and decreased in 4 cases (27\%).

Survival analysis revealed a median progression free survival (PFS) of 6.3 months and PFS at six months of $47 \%$. The survival at one year is $33.3 \%$, and $6.7 \%$ at ten years. The median survival is 8.54 months $(95 \% \mathrm{CI}=$ 4.0 - 13.5). The Kaplan-Meier survival curve is shown in Figure 3.

In accordance with other Phase II studies conducted at the initiation of this trial, the possible responses to the 


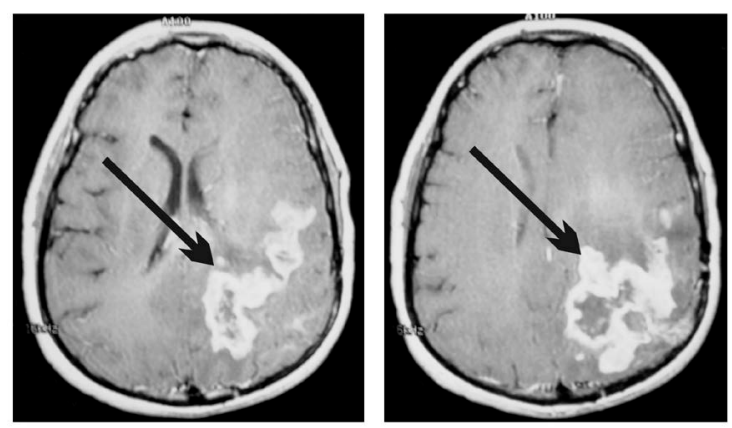

A. Baseline, March 14, 1996

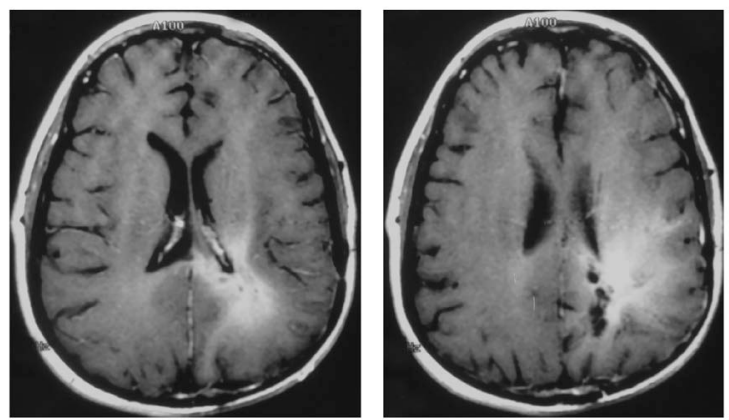

B. MRI, March 17, 1997

Figure 1. Patient 3. 17-year-old diagnosed with anaplastic astrocytoma which reoccurred after subtotal resection, standard radiation therapy and chemotherapy with BCNU. Started ANP on March 19, 1996. Contrast-enhanced MRIs: A-Baseline, B-Complete Response.

treatment were CR, PR, SD and PD. CR required the disappearance of all enhancing lesions, sustained for at least 4 weeks, and only physiologic replacement doses of steroids were acceptable. PR required $50 \%$ or higher decrease of the sum of the products of the two largest perpendicular diameters of enhancing lesions and stable or reduced corticosteroid doses. PD was determined when there was over $50 \%$ increase of enhancing lesions or new lesions, and SD was the status between PR and PD. The results of all MRI and PET scans were verified by radiologists not affiliated with BRI or BC and their determination of response was accepted. Study subjects were categorized by their overall best response during the course of the treatment. The duration of each response was measured from the date that the criteria for the outcome were first met until the date that PD was first documented. The original protocol required more than $50 \%$ increase of tumor size for PD, but in the final analysis, a $25 \%$ increase was set. All cases reported in this paper were re-evaluated by RANO criteria to allow comparison with other studies. In the case of SD, the duration was measured from the time therapy commenced.

During the study, the generally accepted criteria for evaluation of responses in high-grade glioma changed toward reliance on OS and PFS rather than tumor responses. As a result, the protocol was amended to include also a survival analysis.

A group of 4/15 patients demonstrated a CR or PR, which is a sufficient number of successful cases needed to show that there is adequate antitumor activity for initiating Phase III testing. Two of these patients achieved CR. The patient diagnosed with AT/RT was determined to have CR by one radiology consultant and PR by another. However, after she died from pneumonia, the autopsy confirmed CR.

The number of patients was in the range of the other studies for recurrent HGG conducted at the same time and is comparable to similar studies conducted in this patient population. The slow accrual rate of patients to this study resulted in a longer than expected study duration. In addition the decision was taken by the investigator to extend the study evaluation/observation period time once 4 patients achieved an objective response (CR/PR).

\section{Safety and Adverse Events}

Safety assessments were analyzed based upon the total number of enrolled patients in the study $(n=19)$. Intense 


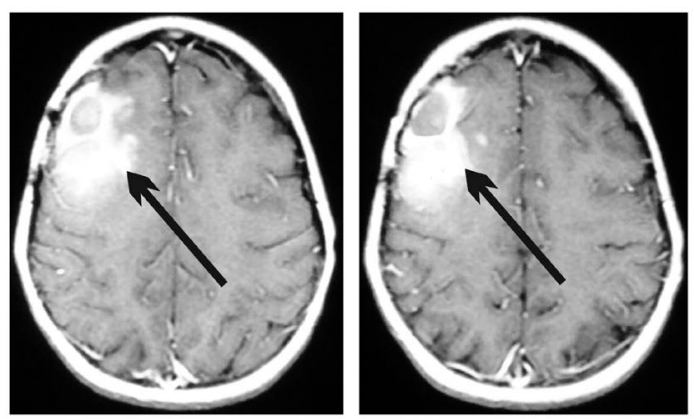

A. Baseline, October 25, 2002

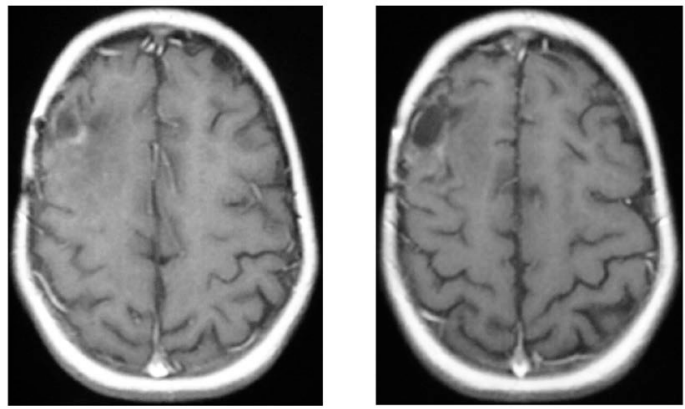

B. MRI, December 30, 2002

Figure 2. Patient 12. 11-year-old female diagnosed with glioblastoma multiforme which reoccurred after two resections, standard radiation therapy and chemotherapy. Started ANP on October 29, 2002. Contrast-enhanced MRIs: ABaseline, B-Partial Response.

Table 3. Phase II clinical studies in recurrent or progressive high-grade gliomas in children.

\begin{tabular}{|c|c|c|c|c|c|c|c|c|c|c|c|c|c|c|}
\hline \multirow{3}{*}{ Author/Treatment } & \multirow{3}{*}{$\begin{array}{c}\text { Patients } \\
\text { Total } \\
\text { No. }\end{array}$} & \multicolumn{2}{|c|}{ Prior Treatment } & \multicolumn{3}{|c|}{$\begin{array}{l}\text { Efficacy } \\
\text { Response }\end{array}$} & \multicolumn{8}{|c|}{ Survival } \\
\hline & & \multirow{2}{*}{$\begin{array}{l}\text { Radiation } \\
\text { Therapy \% }\end{array}$} & \multirow[b]{2}{*}{ Chemotherapy \% } & \multirow[b]{2}{*}{ CR } & \multirow[b]{2}{*}{ PR } & \multirow[b]{2}{*}{ SD } & \multicolumn{2}{|c|}{ PFS } & \multicolumn{6}{|c|}{ OS (\%) } \\
\hline & & & & & & & $\begin{array}{c}\text { Median } \\
(\text { mos) }\end{array}$ & $6 \mathrm{mos}$ & $\begin{array}{c}\text { Median } \\
\text { (mos) }\end{array}$ & $1 \mathrm{yr}$ & 2 yrs & 3 yrs & 5 yrs & $10 \mathrm{yrs}$ \\
\hline $\begin{array}{l}\text { [15] Lashford et al. } \\
\text { 2002, } \\
\text { Temozolomide }\end{array}$ & 33 & All & 33 & 0 & 12 & 18 & & & 4.7 & & & & & \\
\hline $\begin{array}{l}\text { [16] Dreyer et al. } \\
\text { 2003, Idarubicin }\end{array}$ & 18 & 88 & 89 & 0 & 5 & 17 & & & & & & & & \\
\hline $\begin{array}{l}\text { [17] Warren et al. } \\
\text { 2006, Lobradimil and } \\
\text { Carboplatin }\end{array}$ & 9 & 95 & 60 & 0 & 0 & 0 & & & & & & & & \\
\hline $\begin{array}{l}\text { [18] Fouladi et al. } \\
\text { 2007, Tipifarnib }\end{array}$ & 38 & $\begin{array}{r}\text { Recurren } \\
\text { high-gr }\end{array}$ & $\begin{array}{l}\text { th or progressive } \\
\text { rade gliomas }\end{array}$ & 0 & 3 & & & & & & & & & \\
\hline $\begin{array}{l}\text { [19] Gururangan et al. } \\
\text { 2010, Bevacizumab } \\
\text { and Irinotecan }\end{array}$ & 18 & All & & 0 & 0 & 44 & 4.2 & 42 & & & & & & \\
\hline $\begin{array}{l}\text { [20] Nicholson et al. } \\
\text { 2007, Temozolomide }\end{array}$ & 23 & 84 & ALL & 0 & 4 & 30 & & & & & & & & \\
\hline $\begin{array}{c}\text { [21] Warren et al. } \\
\text { 2012, } \\
\text { 06-benzylguanine }\end{array}$ & 25 & ALL & ALL & 0 & 4 & 16 & & 16 & & & & & & \\
\hline $\begin{array}{l}\text { Burzynski et al. 2014, } \\
\text { Antineoplastons } \\
\text { A10 and AS2-1 }\end{array}$ & 15 & 80 & 93 & 13 & 13 & 20 & 6.3 & 53 & 8.4 & 33.3 & 6.7 & 6.7 & 6.7 & $6.7^{*}$ \\
\hline
\end{tabular}

CR—complete response, PR—partial response, SD—stable disease, PFS—progression-free survival, OS—overall survival, D—No information available on prior treatment. "One patient survived 10 years less 7 days from start of ANP therap. 


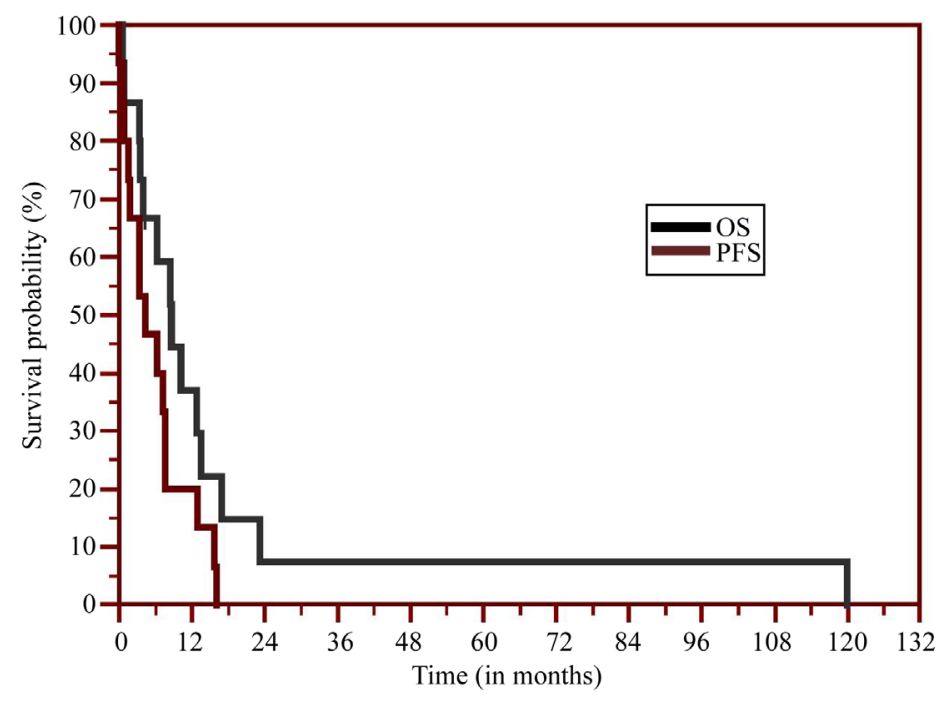

Figure 3. The Kaplan-Meier survival curve.

monitoring of patient safety was conducted during the first two months of therapy and involved daily direct questioning concerning adverse events, first at the clinic and then followed by daily phone calls during the home administration phase. After two months, telephone contact was conducted on a weekly basis. Adverse events were categorized according to CTCAE v3, and compared to other studies (see Table 4). No long-term ADEs to ANP were reported. Brain tumor patients frequently receive corticosteroids as part of their therapeutic regimen to reduce cerebral edema around tumors. The use of corticosteroids, the infusion of large volumes of sodiumcontaining solutions during ANP therapy, and the brain tumor itself predispose a patient to an increased incidence of serum sodium concentration abnormalities. As a result, Grade 3 and Grade 4 reversible hypernatremia was reported in two cases (11\%). Grade 3 reversible neutropenia was found in 5\% of cases.

\section{Discussion}

Recent clinical trials in pediatric HGG were extensively reviewed [4] [6] [9]. The results of these studies, even those carried out with combination treatment regimens showed that efficacy did not improve beyond standard radiation therapy which remains the accepted treatment. Less than $10 \%$ of children with HGG do not survive more than two years from diagnosis, and very few live beyond five years. Only seven trials have been conducted recently in mixed populations of pediatric patients. Most patients in these trials were diagnosed with HGG although a percentage had diagnoses of DIPG. Lashford et al. reported data on the treatment of 33 patients diagnosed with HGG using radiation therapy and TMZ [15]. They reported PR of $12 \%$ and SD of $18 \%$, and a median survival of 4.7 months. Dreyer et al. treated 18 patients with recurrent or progressive disease after conventional RT and chemotherapy [16]. The patients were treated with idarubicin, an analogue of daunomycin. A PR of $5 \%$ and $17 \%$ SD was reported. Warren et al. used a combination of lobradimil and carboplatin to treat 9 patients at first relapse [17]. There were no objective responses, and median time to progression was only 2.6 months. Tipifarnib was used by Fouladi et al. in a Phase II trial for recurrent or progressive HGG including DIPG and PNET [18]. In 38 patients there was one PR (3\%). Gururangan et al. used a combination of bevacizumab and irinotecan in children with recurrent malignant glioma [19]. In the population of 18 patients there were no ORs, but $44 \%$ SD. Median PFS was 4.2 months, and six month PFS was $42 \%$. Nicholson et al. in a study involving 23 children reported 4\% PR and 30\% SD with no survival data [20]. Warren, et al. conducted a Phase II study of 06-benzylguanine and temozolomide in the recurrent pediatric HGG and brainstem gliomas in 25 patients [21]. They reported $4 \%$ of PR and $16 \%$ SD with PFS of $16 \%$ at six months. These trials failed to prove efficacy and the treatments were associated with substantial toxicity (see Table 4).

The results of our trial compare favorably to the other studies with 13\% of each of CR and PR. The median PFS of 6.3 months and PFS at six months of 53\% is higher than in any of the other reported studies. The other studies do not report the survival data in excess of one year, because OS of the patients was very short. In our study, $33.3 \%$ of patients were alive at one year and $6.7 \%$ survived 10 years. 
Table 4. Incidence of adverse drug experiences (ADE), Grades 3 and 4, reported in antineoplastons treatment compared to the studies of chemotherapy for high-grade gliomas.

\begin{tabular}{|c|c|c|c|c|c|c|}
\hline ADE (Incidence \%) & $\begin{array}{l}\text { Burzynski et al. } \\
2014 \\
\text { Antineoplastons }\end{array}$ & $\begin{array}{c}\text { [15] Lashford et } \\
\text { al. } 2002 \\
\text { Temozolomide }\end{array}$ & $\begin{array}{l}\text { [16] Dreyer et al. } \\
2003 \text { Idarubicin }\end{array}$ & $\begin{array}{l}\text { [17] Warren et al. } \\
2006 \text { Lobradimil } \\
\text { and Carboplatin }\end{array}$ & $\begin{array}{l}\text { [18] Fouladi et al. } \\
2007 \text { Tipifarnib }\end{array}$ & $\begin{array}{l}\text { [19] Gururangan et } \\
\text { al. } 2010 \\
\text { Bevacizumab and } \\
\text { Irinotecan }\end{array}$ \\
\hline Hypokalemia & & & & & 1 & \\
\hline Hypernatremia & 11 & & & & 1 & \\
\hline Fatigue & & 1 & & & & 7 \\
\hline Thrombocytopenia & & 7 & 53 & 42 & 15 & 13 \\
\hline Neutropenia & 5 & & 82 & 49 & 18 & \\
\hline Leukopenia & & 5 & & & 15 & \\
\hline Lymphopenia & & & & & 4 & \\
\hline Anemia & & & & 12 & 4 & \\
\hline Nausea & & & & 2 & 3 & \\
\hline Vomiting & & 2 & & 2 & 2 & \\
\hline Anorexia & & $<1$ & & & 3 & \\
\hline Infection & & & 16 & & 5 & \\
\hline Sepsis & & 2 & 3 & & & \\
\hline Pneumonia & & 2 & 2 & & & \\
\hline Fever & & & & & 1 & \\
\hline Neuropathy & & $<1$ & & & 2 & \\
\hline Cerebral ischemia & & & & & 2 & 7 \\
\hline Hemorrhage & & & 2 & & & \\
\hline Hypertension & & & & & 1 & 27 \\
\hline Cardiotoxicity & & & 1 & & & 13 \\
\hline Nephrotoxicity & & & 1 & 2 & & \\
\hline Ototoxicity & & & & 2 & & \\
\hline Ocular toxicity & & & & & 1 & \\
\hline Stomatitis & & & 1 & & & \\
\hline Pruritus & & & & & 1 & \\
\hline Altered mental status & & & & & 1 & \\
\hline Seizures & & $<1$ & & 2 & 2 & \\
\hline Headache & & & & 5 & 1 & \\
\hline Pain & & $<1$ & & 2 & 1 & \\
\hline Hepatotoxicity & & & & & & 13 \\
\hline Hypermagnesemia & & & & & 1 & \\
\hline Weight loss & & & & & 1 & \\
\hline Syncope & & & & 2 & 1 & \\
\hline Lipase & & & & & 1 & \\
\hline
\end{tabular}




\section{Conclusion}

The treatment of recurrent high-grade and brainstem glioma create the greatest challenge for pediatric neurooncologists. This small Phase II study of ANP A10 and AS2-1 reported encouraging CR and PR in recurrent atypical teratoid/rhabdoid tumor, glioblastoma multiforme, and anaplastic astrocytoma with encouraging survival data. Additional Phase II studies of ANP in pediatric high-grade glioma and brainstem glioma as well as astrocytoma and optic pathway glioma have been completed and manuscripts are currently in development.

\section{Acknowledgements}

The authors express their appreciation to the additional physicians involved in the care of the patients: Robert A. Weaver, M.D., Robert I. Lewy, M.D., Eva Kubove, M.D., Barbara Szymkowski, M.D., and Mohammad Khan, M.D. Preparation of the manuscript was provided by Carolyn Powers. Editorial assistance was provided by Malcolm Kendrick, M.D.

\section{Conflict of Interest}

All authors are employed by Burzynski Clinic. Dr. Stanislaw R. Burzynski and Dr. Gregory S. Burzynski are shareholders and directors, and Dr. Tomasz J. Janicki is the vice-president of Burzynski Research Institute, Inc. Dr. Stanislaw R. Burzynski is President of Burzynski Research Institute, Inc. and Dr. Gregory Burzynski is Vice President of Burzynski Research Institute, Inc.

\section{References}

[1] Van Meir, E.G., Hadjipanayis, C.G., Norden, A.D., Shu, H.K., Wen, P.Y. and Olson, J.J. (2010) Exciting New Advances in Neuro-Oncology: The Avenue to a Cure for Malignant Glioma. Cancer Journal for Clinicians, 60, 166-193. http://dx.doi.org/10.3322/caac.20069

[2] Stupp, R., Mason, W.P., van den Bent, M.J., Weller, M., Fisher, B., et al. (2005) Radiotherapy plus Concomitant and Adjuvant Temozolomide for Glioblastoma. The New England Journal of Medicine, 352, 987-996. http://dx.doi.org/10.1056/NEJMoa043330

[3] Wick, W., Weller, M., Weiler, M., Batchelor, T., Yung, A.W. and Platten, M. (2011) Pathway Inhibition: Emerging Molecular Targets for Treating Glioblastoma. Neuro-Oncology, 13, 566-579. http://dx.doi.org/10.1093/neuonc/nor039

[4] Finlay, J.L. and Zacharoulis, S. (2005) The Treatment of High Grade Gliomas and Diffuse Intrinsic Pontine Tumors of Childhood and Adolescence: A Historical and FuturisticPerspective. Journal of Neuro-Oncology, 75, 253-266. http://dx.doi.org/10.1007/s11060-005-6747-7

[5] CBTRUS (2013) CBTRUS Statistical Report Supplement 2013: Primary Brain and Central Nervous System Tumors Diagnosed in the United States 2006-2010. Neuro-Oncology, 15, ii1-ii56.

[6] Burzynski, S.R. (2004) The Present State of Antineoplaston Research (1). Integrative Cancer Therapies, 3, 47-58. http://dx.doi.org/10.1177/1534735403261964

[7] Burzynski, S.R., Kubove, E. and Burzynski, B. (1992) Phase II Clinical Trials of antineoplastons A10 and AS2-1 Infusions in Astrocytoma. In: Adam, D., Ed., Recent Advances in Chemotherapy, Futuramed Publishers, Munich, 25062507.

[8] Hawkins, M.G. and Friedman, M.A. (1992) National Cancer Institute's Evaluation of Unconventional Cancer Treatments. Journal of the National Cancer Institute, 84, 1699. http://dx.doi.org/10.1093/jnci/84.22.1699

[9] Burzynski, S.R. (2006) Treatments for Astrocytic Tumors in Children: Current and Emerging Strategies. Pediatric Drugs, 8, 167-168. http://dx.doi.org/10.2165/00148581-200608030-00003

[10] Burzynski, S.R., Janicki, T.J., Burzynski, G.S. and Marszalek, A. (2014) The Response and Survival of Children with Recurrent Diffuse Intrinsic Pontine Glioma Based on Phase II Study of Antineoplastons A10 and AS2-1 in Patients with Brainstem Glioma. Child’s Nervous System. http://link.springer.com/article/10.1007/s00381-014-2401-z

[11] Burzynski, S.R., Janicki, T.J., Burzynski, G.S. and Marszalek, A. (2013) Long-Term Survival (>13 Years) in a Child with Recurrent Diffuse Intrinsic Pontine Glioma: A Case Report. Journal of Pediatric Hematology/Oncology, http://journals.lww.com/jpho-online/Abstract/publishahead/Long_term_Survival__13_Years_in_a_Child_With.9899 7.aspx http://dx.doi.org/10.1097/MPH.0000000000000020

[12] Chang, S.M., Kuhn, J.G., Robins, H.I., Schold, S.C., Spence, A.M., Berger, M.S., Mehta, M.P., Bozik, M.E., Pollack, I., Schiff, D., Gilbert, M., Rankin, C. and Prados, M.D. (1999) Phase II Study of Phenylacetate in Patients with Recurrent 
Malignant Flioma: A North American Brain Tumor Consortium Report. Journal of Clinical Oncology, 17, 984-990.

[13] Wen, P.Y., Macdonald, D.R., Reardon, D.A., Cloughesy, T.F., Sorensen, A.G., et al. (2010) Updated Response Assessment Criteria for High-Grade Gliomas: Response Assessment in Neuro-Oncology Working Group. Journal of Clinical Oncology, 28, 1963-1972. http://dx.doi.org/10.1200/JCO.2009.26.3541

[14] Weller, M., Cloughesy, T., Perry, J.R. and Wick, W. (2013) Standards of Care for Treatment of Recurrent Glioblastoma-Are We There Yet? Neuro-Oncology, 15, 4-27. http://dx.doi.org/10.1093/neuonc/nos273

[15] Lashford, L.S., Thiesse, P., Jouvet, A., Jaspan, T., Couanet, D., Griffiths, P.D., Doz, F., Ironside, J., Robson, K., Hobson, R., Dugan, M., Pearson, A.D.J., Vassal, G. and Frappaz, D. (2002) Temozolomide in Malignant Gliomas of Childhood: A United Kingdom Children's Cancer Study Group and French Society for Pediatric Oncology Intergroup Study. Journal of Clinical Oncology, 20, 4684-4691. http://dx.doi.org/10.1200/JCO.2002.08.141

[16] Dreyer, Z.E., Kadota, R.P., Stewart, C.F., Friedman, H.S., Mahoney, D.H., et al. (2003) Phase 2 Study of Idarubicin in Pediatric Brain Tumors: Pediatric Oncology Group Study POG 9237. Neuro-Oncology, 5, 261-267. http://dx.doi.org/10.1215/S115285170200056X

[17] Warren, K., Jakacki, R., Widemann, B., Aikin, A., Libucha, M., et al. (2006) Phase II Trial of Intravenous Lobradimil and Carboplatin in Childhood Brain Tumors: A Report from the Children's Oncology Group. Cancer Chemotherapy and Pharmacology, 58, 343-347. http://dx.doi.org/10.1007/s00280-005-0172-7

[18] Fouladi, M., Nicholson, H.S., Zhou, T., Laningham, F., Helton, K.J., et al. (2007) A Phase II Study of the Farnesyl Transferase Inhibitor, Tipifarnib, in Children with Recurrent or Progressive High-Grade Glioma, Medulloblastoma/ Primitive Neuroectodermal Tumor, or Brainstem Glioma: A Children's Oncology Group Study. Cancer, 110, 25352541. http://dx.doi.org/10.1002/cncr.23078

[19] Gururangan, S., Chi, S.N., Poussaint, T.Y., Onar-Thomas, A., Gilbertson, R.J., et al. (2010) Lack of Efficacy of Bevacizumab Plus Irinotecan in Children with Recurrent Malignant Glioma and Diffuse Brainstem Glioma: A Pediatric Brain Tumor Consortium Study. Journal of Clinical Oncology, 28, 3069-3075. http://dx.doi.org/10.1200/JCO.2009.26.8789

[20] Nicholson, H.S., Kretschmar, C.S., Krailo, M., Bernstein, M., Kadota, R., et al. (2007) Phase 2 Study of Temozolomide in Children and Adolescents with Recurrent Central Nervous System Tumors. A Report from the Children's Oncology Group. Cancer, 110, 1542-1550. http://dx.doi.org/10.1002/cncr.22961

[21] Warren, K.E., Gururangan, S., Geyer, J.R., McLendon, R.E., Poussaint, T.Y., et al. (2012) A Phase II Study of 06-Benzylguanine and Temozolomide in Pediatric Patients with Recurrent or Progressive High-Grade Gliomas and Brainstem Gliomas: A Pediatric Brain Tumor Consortium Study. Journal of Neuro-Oncology, 106, 643-649. http://dx.doi.org/10.1007/s11060-011-0709-z 